\title{
The effects of sterol mutants of the yeast Saccharomyces cerevisiae on the outcome of competition between Drosophila melanogaster and $D$. simulans
}

\author{
M. Bos, A. Boerema \& G. Lammers \\ Department of Genetics, University of Groningen, P.O. Box 14, 9750 AA Haren. The Netherlands
}

\begin{abstract}
The effect of different yeast variants of the yeast Saccharomyces cerevisiae on the outcome of competition between Drosophila simulans and D. melanogaster was investigated. In all experiments differential birth rate was the effective mode of species competition. Addition of live yeast and especially mixtures of yeast strains improved the competition situation of a species, but could not prevent the extinction.
\end{abstract}

\section{Introduction}

In natural populations species are associated with each other and may compete for limited resources. The sibling species Drosophila melanogaster and D. simulans are sympatric and utilize yeasts for their essential foodcomponents (Sang, 1978; review). It has been claimed that genetic variability in Drosophila species is correlated with changes in the yeast environment (Powell, 1971; McDonald \& Ayala, 1974; Bos et al., 1976; Kircher, 1969). In view of the importance of yeasts in the nutrition of Drosophila and the differential attractiveness of different yeasts to sympatric species (Dobzhansky et al., 1956), one might expect the relative competitive abilities of the species in laboratory populations to vary depending on the yeasts used in the culture (see for review of interspecific competition: Barker, in prep.). The abilities may sometimes be influenced by the composition of yeast species present in the culture as shown by El-Helw and Ali (1970) and El-Helw et al. (1972). Bos et al. (1976) found that species of Drosophila differ in their ability to utilize ergosterol-deficient mutant strains of the yeast Saccharomyces cerevisiae. In monoxenic cultures containing two of these mutant yeasts (erg-2 and erg-3) the survival of $D$. simulans is superior to that of $D$. melanogaster. One might expect from this that fixation of D. melanogaster as found in so many mixed populations of the two species (Moore, 1952; Tantawy \& Solimar, 1967; Barker, in prep.), can at least be postponed in an environment which includes these sterol-mutant yeast strains. We report here the results of such competition experiments on mixtures of yeast strains with different sterol contents and discuss the effects of medium on interspecific competition.

\section{Material and methods}

\section{Yeast strains}

The yeast strains used in these experiments were a wildtype strain of Saccharomyces cerevisiae (A $18.4 \mathrm{~A})$, two sterol mutants derived from this stock (erg-2 and erg-3; formerly named pol mutants in Molzahn \& Woods, 1972) and a commercial wildtype strain, originally bought as active dry yeast. The strains were grown in 2 litres Erlenmeyer flasks on a rotary shaker at $28^{\circ} \mathrm{C}$ in $750 \mathrm{ml}$ of yeast complete medium (Molzahn \& Woods, 1972). 
Drosophila stocks

Flies used for the experiments were taken from large laboratory massbred populations, maintained under a system of random mating since 1975 . The melanogaster population derived from a two-way cross between Oregon and Novosibirsk strains, homozygous for the body colour mutation ebony. The simulans populations derived from an F2 of a cross between a Sheffield massbred strain and a white-eyed strain from Groningen. The population was homozygous for the eye colour mutant white eye. Culture temperature was always $25^{\circ} \mathrm{C}$.

\section{Experimental procedures}

\section{Competition experiments}

Competition was studied in four different experiments. Two series of Cage ( $A$ and $B$ ) and two series of Bottle experiments (C and D).

Cage experiments A. Eight types of cages were started november 1976, each with 1000 flies ( 250 우 and $250 \hat{\partial} \hat{\delta}$ from each of the species) and stopped after five months. The types of the cages are listed in Table 1.

Table 1. Types of cages used.

\begin{tabular}{rcccc}
\hline Cages & $\begin{array}{c}\text { Yeasts } \\
\text { wildtype } \\
\text { (A18.4A) }\end{array}$ & erg-2 & erg-3 & $\begin{array}{l}\text { Standard* } \\
\text { medium }\end{array}$ \\
\hline Al & $100 \%$ & - & - & - \\
2 & - & $100 \%$ & - & - \\
3 & - & - & $100 \%$ & - \\
4 & - & - & - & $100 \%$ \\
5 & $80 \%$ & $20 \%$ & - & - \\
6 & $20 \%$ & $80 \%$ & - & - \\
7 & $80 \%$ & - & $20 \%$ & - \\
8 & $20 \%$ & - & $80 \%$ & - \\
\hline
\end{tabular}

* 'standard medium' $=1000 \mathrm{ml}$ water, $19 \mathrm{~g}$ agar, $32 \mathrm{~g}$ dead wildtype yeast, $54 \mathrm{~g}$ sucrose and $13 \mathrm{ml}$ nipagin solution $(10 \mathrm{~g}$ nipagin in $100 \mathrm{ml}$ ethanol, $96 \%$ ).

The environments are here identified by the percentage of mutant yeast they contain (e.g. 20\% erg-2).

Live yeast was offered on the surface of an agar gel $(2 \%)$ in food cups, different yeasts in different cups. Every cup contained about $2 \mathrm{~g}$ of live yeast. Cups were replaced according to a scheme, which guaranteed that some fresh cups were provided every two days and that a cup stayed about 17 days in a cage. Sampling of eggs to estimate the proportion of the two species was done regularly (once a month) by inserting five fresh cups in each cage for one day. The emerging adults were collected and scored. Each type of cage was duplicated.

Cage experiment $B$. Experiment A was repeated between October 1977 and January 1978, because (1) in some cages contamination with fertile, wild type melanogaster was observed, although replicates with and without contamination reached the same final outcome - the fixation of melanogaster; (2) we wanted to test whether our sampling technique was reliable for estimating the proportion of the species present in the cages. In this experiment eggs were collected on slices of standard medium placed on corks in the cages. Slices with eggs were placed in standard food bottles and the number of flies hatching from these bottles was then determined. During the egg-collection period the number of flies visiting the slices was also counted, affording a second measure of the frequency of the species. Flies were counted $1 \mathrm{~h}$, and $2 \mathrm{~h}$, after insertion of the slices and the two counts were averaged.

Bottle experiment $C$. Experiment $\mathrm{C}$ was done in bottles, started in April 1978 and lasted five generations. Each bottle was supplemented with $5 \mathrm{~g}$ of live yeast on top of $30 \mathrm{ml}$ of sucrose agar $(5.4 \%$ sucrose; $2 \%$ agar). If a bottle was supplemented with two yeasts, they were added separately. The eight types of food medium were assayed ( $\mathrm{Cl}-8)$, with only one difference: the wildtype yeast was the commercial live yeast. A population on a medium consisted of four bottles with flies, started with 20 pairs of each of the two species per bottle. After two weeks the hatched flies were scored as simulans or melanogaster and then divided into four fresh bottles for about one day of egg-laying. An advantage of these experiments with non-overlapping generations was among others the fact that the population size and species frequencies could be determined every generation.

In addition to the eight types of media the effect of live yeast on top of standard medium was assayed. 
Bottle experiment $D$. A large-scale bottle experiment was carried out during the winter of 1978 1979 for six generations. Two replicates of every experiment (D1-8) were used. For three generations all flies were counted and then used to start the next generation, but from generation four onwards every fresh bottle was started with 40 pairs of flies using the same frequencies as in the total population. In addition an environment containing $95 \%$ erg-3 yeast was assayed.

\section{Other procedures in the D experiment}

Production of adults. The production of offspring by females was measured using vials ( 4 우, 3-5 days old) containing one of both species. Thirty replicates were made using standard medium (see experiment A) with both species together. On wildtype yeast ( $1 \mathrm{~g}$ live yeast on $9 \mathrm{ml}$ of $2 \%$ agar) there were seven replicates for $D$. melanogaster, and twelve replicates for $D$. simulans. Egglaying time was $24 \mathrm{~h}$.

Development time. Measurements were made using twice-daily counts of hatching adult flies in bottles and the time half way egglaying period. Pure species cultures were given a short egglaying time ( $8 \mathrm{~h} ; 6-10$ replicate bottles), mixed species cultures a long egglaying time ( $20 \mathrm{~h} ; 6$ replicates) to measure egg-to-adult-development time in 'optimal' and competitive conditions.

Body weight. In the same experiment body weight was determined by weighing five groups of five males from each environment.

\section{Results}

\section{Competition and yeast environments}

The course of the proportion of D. simulans in competition with $D$. melanogaster in two cage experiments ( $\mathrm{A}$ and $\mathrm{B}$ ) and two bottle experiments (C and D) is presented in Figure 1. Table 2 presents population sizes in experiments $B$ and $D$ after two months of competition. All sample periods are included. From these data five points are obvious:

(1) In all the environments in experiments $\mathrm{A}, \mathrm{C}$ and $\mathrm{D}$ the final result was extinction of Drosophila simulans.

(2) In experiments B all the populations were fixed in D. simulans or had a high frequency, except on the 'standard' medium. These exceptional results of experiment $B$ will be analyzed in part 2 of the results.

(3) On erg-2 and erg-3 medium both species became extinct in all experiments except the $B$ cages and one A replicate.

(4) The decrease of the frequency of $D$. simulans was gradually on live wildtype yeast and the mixtures of live yeasts. On standard medium with killed yeast the decrease went much faster: e.g. the proportion of simulans in cages decreased from $50 \%$ to about $3 \%$ (9 angles) in 1 month. On live yeast the proportion was still at least 25\% (30 angles) after one month of competition, which is about 1 or 2 generations (Fig. 1a). The use of live yeast apparently postponed the extinction of simulans.

The effect of addition of live yeast to the standard medium was measured in a separate experiment which was performed simultaneously with experiment $D$. The results are presented in Figure 2. These data confirmed the above conclusion that live yeast postponed the extinction of $D$. simulans.

(5) Since within experiments and even between experiments $\mathrm{A}, \mathrm{C}$ and $\mathrm{D}$ there was a good correspondence between replicates on the same medium, we calculated the average proportions of D. simulans (angles), averaged over experiments $A, C$ and $D$ after one and two months of competion ( $\overline{\mathrm{x}} \pm$ s.e.) (Table 3 ).

Table 2. Population size (average of 2-4 replicates) in experiments B (cages) and D (bottles), two months after the start of competition.

\begin{tabular}{|c|c|c|c|c|c|c|c|c|}
\hline & \multicolumn{8}{|c|}{ Yeast environment } \\
\hline & $\begin{array}{c}1 \\
100 \% \\
+- \text { yeast }\end{array}$ & $\begin{array}{c}2 \\
100 \% \\
\text { erg- } 2\end{array}$ & $\begin{array}{c}3 \\
100 \% \\
e r g-3\end{array}$ & $\begin{array}{l}4 \\
\text { standard }\end{array}$ & $\begin{array}{c}5 \\
20 \% \\
e r g-2\end{array}$ & $\begin{array}{l}6 \\
80 \% \\
e r g-2\end{array}$ & $\begin{array}{c}7 \\
20 \% \\
e r g-3\end{array}$ & $\begin{array}{c}8 \\
80 \% \\
\text { erg- } 3\end{array}$ \\
\hline Exp. B & 396 & 77 & 326 & 252 & 407 & 188 & 543 & 445 \\
\hline Exp. D & 213 & 0 & 0 & 297 & 515 & 397 & 591 & 459 \\
\hline
\end{tabular}


$\begin{array}{ll}\text { (1) } 100 \%+\text {-yeast } & \text { (2) } 100 \% \text { erg- } 2\end{array}$
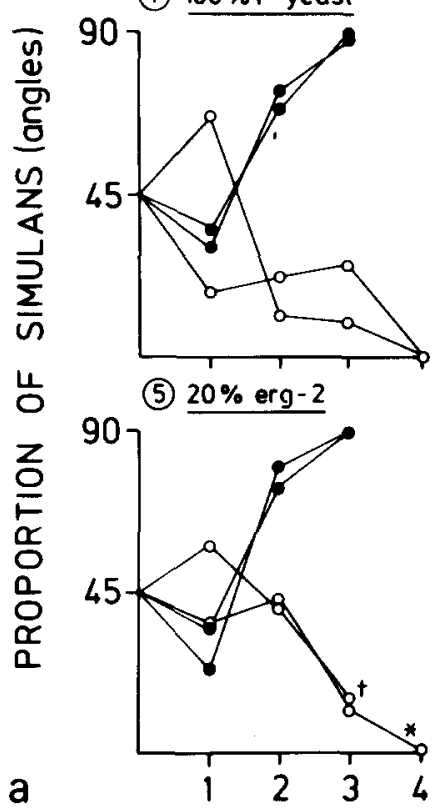

(1) $100 \%+$-yeast

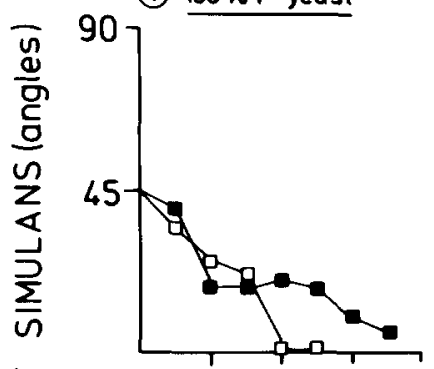

$\frac{1}{0}$

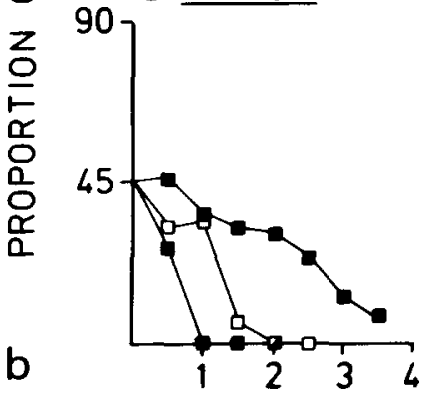

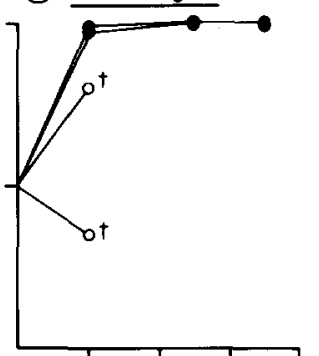

(6) $80 \% \mathrm{erg}-2$

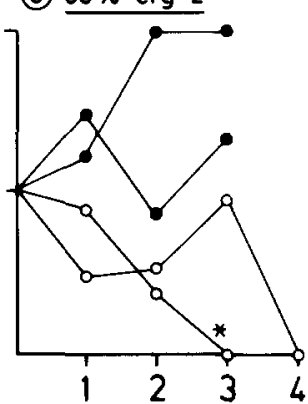

(2) $100 \%$ erg -2

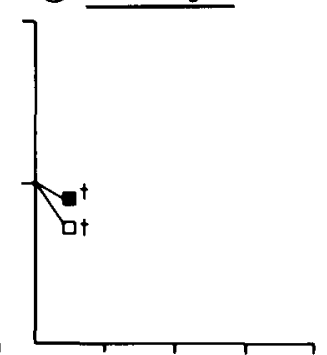

(6) $80 \%$ erg -2

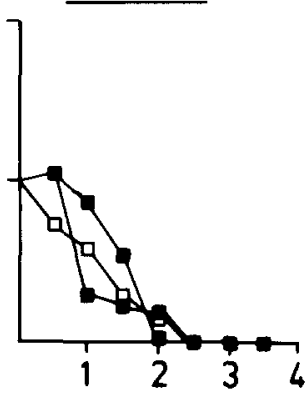

(3) $100 \%$ erg -3

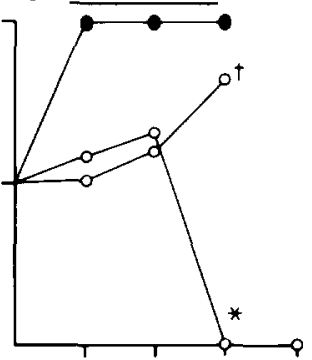

(7) $20 \%$ erg -3

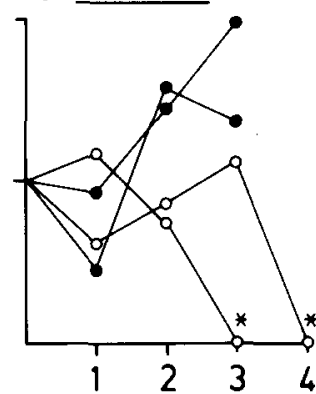

(3) $100 \%$ erg - 3

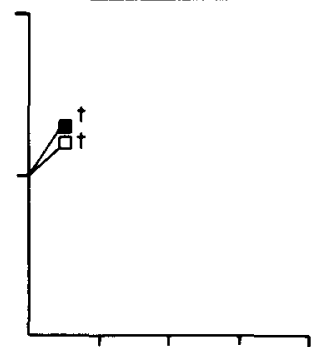

(7) $20 \%$ erg -3

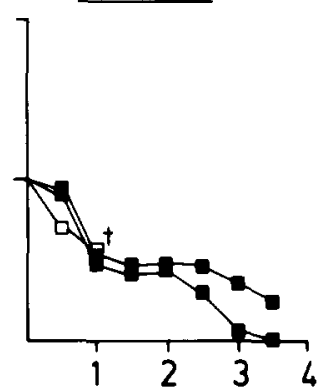

(4) Standard

a $\rightarrow$ A replicates

$\longrightarrow$ B. replicates

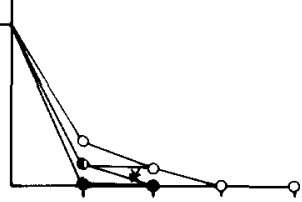

(B) $80 \%$ erg -3

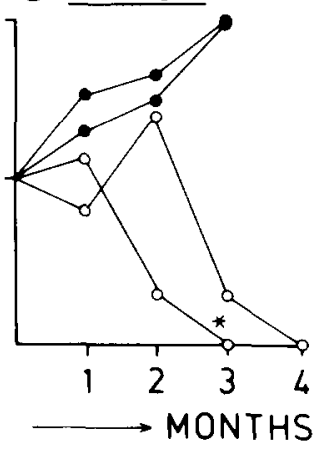

(4) Standard

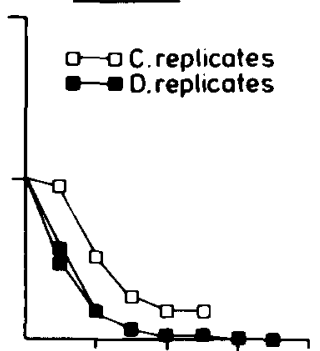

(8) $80 \% \operatorname{erg}-3$

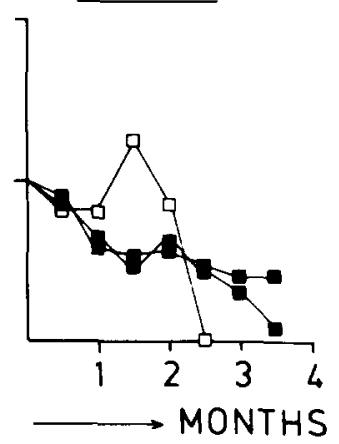

Fig. 1. Proportion of D. simulans in competition: (a) Cages, * cages contaminated with D. melanogaster; (b) Bottles.

Table 3. Average proportions of D. simulans after one and two months of competition (exps A, C and D).

\begin{tabular}{rrrlllr}
\hline Mo. Standard & $80 \%$ erg- & $100 \%+$ - yeast & $20 \%$ erg -2 & $20 \%$ erg-3 & $80 \%$ erg-3 \\
\hline 1 & $11.2 \pm 3.0$ & $27.8 \pm 5.1$ & $32.2 \pm 11.8$ & $32.5 \pm 9.2$ & $29.7 \pm 5.7$ & $35.7 \pm 4.3$ \\
2 & $2.8 \pm 1.7$ & $10.8 \pm 4.2$ & $13.4 \pm 5.1$ & $22.8 \pm 9.5$ & $28.2 \pm 4.9$ & $33.4 \pm 8.5$ \\
\hline
\end{tabular}


After one month the proportion of simulans on 'standard' medium is significantly lower ( $t$-test; $P<0.05)$ than the other proportions.

After two months the proportion on 'standard' is still significantly lower $(P<0.05)$ than the other proportions except ' $80 \%$ erg-2'. Comparing all pairs of treatments a difference of 10 units is significant (Tuckey's t-test; $\mathrm{P}<0.05$ ).

These data illustrated again the postponement of simulans extinction on live yeast but they also suggested that after two months of competition $D$. simulans extinction was postponed especially in the 'mixed'-erg-3 environments compared to the standard environment (see also Fig. 1). This is demonstrated also in Figure 3. In this figure the differences between the proportion (average of replicates) of simulans on mixed erg- 3 and $100 \%$ erg- 3 yeast environments and on wildtype yeast are given. A short experiment on $95 \%$ erg-3 is included here. It can be seen that with increasing amounts of erg- 3 the proportion of simulans increased.

\section{The exceptional experiment $B$}

In experiment $B$ all the populations became fixed or had a high frequency for $D$. simulans, except the two replicates on standard medium (Fig. la). This is in contrast with the results in experiments $A$, $\mathrm{C}$ and $\mathrm{D}$, where $D$. melanogaster became fixed.

In experiment $A$ the frequency of a species was determined by counting the numbers of individuals hatching from egg samples taken from the cages. In the case of experiment $B$ frequencies were estimated in the same way, but also directly by counting the number of flies present on the oviposition sites. These 'direct' data are presented in Table 4. The two methods of frequency determination turned out to be the same: the correlation between the two determinations was high and significant $(r=0.92$; $\mathrm{P}<0.001$ ). So the contrasting results reflected the real situations in the cages.

From the counts of adults in the cages and the numbers of offspring in the egg-collection samples the productivity (flies/ ) could be estimated (Table 5).

In the 'standard medium' cages simulans became extinct, in all the other environments melanogaster disappeared. Table 5 shows that in twenty-two out of 32 comparisons a lower production was associated with extinction. In four comparisons we scored

Table 4. Proportion in angles of D. simulans flies, visiting the oviposition sites in the cage populations (experiment B). Two cages ( 1 and 2) of each yeast environment.

\begin{tabular}{|c|c|c|c|c|c|c|c|c|c|c|c|c|c|c|c|c|}
\hline \multirow{5}{*}{$\begin{array}{l}\text { Sampling } \\
\text { period } \\
\text { (month) }\end{array}$} & \multicolumn{16}{|c|}{ Yeast environment } \\
\hline & \multirow{3}{*}{\multicolumn{2}{|c|}{$\begin{array}{c}1 \\
100 \% \\
+ \text {-yeast }\end{array}$}} & \multirow{3}{*}{\multicolumn{2}{|c|}{$\begin{array}{c}2 \\
100 \% \\
\text { erg- } 2\end{array}$}} & \multirow{3}{*}{\multicolumn{2}{|c|}{$\begin{array}{c}3 \\
100 \% \\
\text { erg-3 }\end{array}$}} & \multirow{3}{*}{\multicolumn{2}{|c|}{$\begin{array}{c}4 \\
\text { standard }\end{array}$}} & \multirow{3}{*}{\multicolumn{2}{|c|}{$\begin{array}{c}5 \\
20 \% \\
\text { erg-2 }\end{array}$}} & \multirow{3}{*}{\multicolumn{2}{|c|}{$\begin{array}{c}6 \\
80 \% \\
\text { erg-2 }\end{array}$}} & \multirow{3}{*}{\multicolumn{2}{|c|}{$\begin{array}{c}7 \\
20 \% \\
e r g-3\end{array}$}} & \multirow{3}{*}{\multicolumn{2}{|c|}{$\begin{array}{c}8 \\
80 \% \\
\text { erg-3 }\end{array}$}} \\
\hline & & & & & & & & & & & & & & & & \\
\hline & & & & & & & & & & & & & & & & \\
\hline & 1 & 2 & 1 & 2 & 1 & 2 & 1 & 2 & 1 & 2 & 1 & 2 & 1 & 2 & 1 & 2 \\
\hline 0 & 45 & 45 & 45 & 45 & 45 & 45 & 45 & 45 & 45 & 45 & 45 & 45 & 45 & 45 & 45 & 45 \\
\hline 1 & 20 & 21 & 90 & 90 & 72 & 89 & 27 & 28 & 24 & 27 & 58 & 51 & 35 & 23 & 48 & 44 \\
\hline 2 & 53 & 77 & 90 & 90 & 87 & 90 & 24 & 18 & 73 & 59 & 51 & 86 & 70 & 63 & 57 & 60 \\
\hline 3 & 70 & 90 & 90 & 90 & 90 & 90 & 0 & 0 & 90 & 75 & $73^{*}$ & 90 & 88 & 58 & 80 & 88 \\
\hline
\end{tabular}

* contaminated with $D$. melanogaster wildtype flies

Table 5. Productivity (flies/\&) in D. melanogaster and simulans in experiment B after one and two months.

\begin{tabular}{|c|c|c|c|c|c|c|c|c|c|c|c|c|c|c|c|c|}
\hline \multirow{5}{*}{$\begin{array}{l}\text { Sampling } \\
\text { period } \\
\text { (month) }\end{array}$} & \multicolumn{16}{|c|}{ Yeast environment } \\
\hline & \multicolumn{2}{|l|}{1} & \multicolumn{2}{|l|}{2} & \multicolumn{2}{|c|}{3} & \multicolumn{2}{|c|}{4} & \multicolumn{2}{|c|}{5} & \multicolumn{2}{|l|}{6} & \multicolumn{2}{|l|}{7} & \multicolumn{2}{|l|}{8} \\
\hline & \multirow{2}{*}{\multicolumn{2}{|c|}{$\begin{array}{l}100 \% \\
+ \text {-yeast }\end{array}$}} & \multirow{2}{*}{\multicolumn{2}{|c|}{$\begin{array}{l}100 \% \\
\text { erg- } 2\end{array}$}} & \multirow{2}{*}{\multicolumn{2}{|c|}{$\begin{array}{l}100 \% \\
\text { erg- } 3\end{array}$}} & \multirow{2}{*}{\multicolumn{2}{|c|}{ standard }} & \multirow{2}{*}{\multicolumn{2}{|c|}{$\begin{array}{l}20 \% \\
\text { erg- } 2\end{array}$}} & \multirow{2}{*}{\multicolumn{2}{|c|}{$\begin{array}{l}80 \% \\
\text { erg- } 2\end{array}$}} & \multirow{2}{*}{\multicolumn{2}{|c|}{$\begin{array}{l}20 \% \\
\text { erg- } 3\end{array}$}} & \multirow{2}{*}{\multicolumn{2}{|c|}{$\begin{array}{l}80 \% \\
\text { erg-3 }\end{array}$}} \\
\hline & & & & & & & & & & & & & & & & \\
\hline & 1 & 2 & 1 & 2 & 1 & 2 & 1 & 2 & 1 & 2 & 1 & 2 & 1 & 2 & 1 & 2 \\
\hline 1. $\mathrm{mel}$. & 6.0 & 7.3 & 0 & 0 & 0 & 0 & 1.3 & 1.5 & 1.1 & 3.6 & 1.2 & 0.5 & 8.8 & 5.8 & 2.5 & 4.7 \\
\hline sim. & 10.5 & 12.2 & 0 & 0 & 1.4 & 0.3 & 0.2 & 0.1 & 0.8 & 5.5 & 1.2 & 0.9 & 14.9 & 5.3 & 11.5 & 1.2 \\
\hline 2. mel. & 1.9 & 3.8 & 0 & 0 & 0 & 0 & 7.0 & 6.0 & 1.1 & 0.9 & 6.5 & 0 & 2.5 & 1.5 & 1.5 & 1.2 \\
\hline sim. & 8.2 & 2.5 & 1.0 & 2.6 & 1.3 & 1.4 & 0.3 & 0.3 & 3.4 & 2.5 & $2.0^{*}$ & 5.2 & 1.7 & 3.0 & 5.9 & 1.2 \\
\hline
\end{tabular}

* contaminated with $D$. melanogaster wildtype flies 
no difference in productivity, in six higher production led to extinction.

So, in experiment B productivity of females can have played an important role in the outcome of competition.

Another inexplicable feature in experiment B is the survival of a population after more than one generation on erg- 2 medium. In our bottle experiments $C$ and $D$, our cage experiment $A$ and previous papers (Bos et al., 1976) populations became extinct after one generation. This and the fact that we recently noticed (unpublished) that condition of yeast cultures can have an extreme influence on the survival of Drosophila larvae, suggests that during experiment $\mathbf{B}$ conditions or quality of yeasts differed from conditions during the other experiments. These unknown exceptional conditions offered the advantage for simulans to outlive melanogaster. The possibility that after a period covering about 20 generations the gene pool of our Drosophila stocks also may have changed, can not be excluded.

\section{Fitness characters in experiment $D$}

In experiment $\mathrm{D}$ productivity of females cultured outside and inside competitive situations on wildtype yeast and standard medium was measured (Table 6). Here again we found a positive relation between production of adults and survival in competition: in experiment $D$ the species with the lowest productivity (simulans) decreased in all bottle populations.

Development time was analyzed during this experiment and the relevant data, including significance of differences within species are shown in Table 7. The development time of both species was longer in the mixed species, high-density conditions and longer on standard medium. In the competitive situation $D$. simulans developed slower than melanogaster on standard medium (t-test, $0.05>\mathrm{P}>$

Table 6. Production of adult flies ( $\overline{\mathrm{x}} \pm$ s.e.) per four females in single (1)- and mixed (2)-species cultures (Experiment D).

\begin{tabular}{lllll}
\hline & \multicolumn{2}{l}{ Wildtype yeast } & \multicolumn{2}{l}{ Standard medium } \\
\cline { 2 - 2 } \cline { 5 - 5 } \cline { 5 - 6 } & $(1)$ & & $(1)$ & $(2)$ \\
\hline D. melanogaster & $59.7 \pm 5.3$ & & $61.1 \pm 2.8$ & $64.8 \pm 1.9$ \\
D. simulans & $52.5 \pm 2.8$ & & $42.2 \pm 2.2$ & $42.4 \pm 3.7$ \\
\hline
\end{tabular}

Table 7. Development time (hours) of males under 'Pure species, low density' and 'Mixed species, high density' conditions: average of replicates \pm s.e. (Between brackets, number of replicates and total number of males).

\begin{tabular}{llll}
\hline & Wildtype yeast & Standard medium & $\mathrm{P}$ \\
\hline $\begin{array}{llll}\text { D. melanogaster } \\
\text { Pure, low }\end{array}$ & $\begin{array}{l}208.8 \pm 0.5 \\
(5,187)\end{array}$ & $\begin{array}{l}226 \pm 3.8 \\
(5,150)\end{array}$ & $* * *$ \\
Mixed, high & $253.5 \pm 0.9$ & $258.8 \pm 2.7$ & n.s. \\
& $(5,472)$ & $(6,723)$ & \\
P (t-test) & $* * *$ & $* * *$ & \\
& & & \\
D. simulans & & & \\
Pure, low & $209.4 \pm 2.3$ & $216.3 \pm 1.5$ & $* *$ \\
& $(4,116)$ & $(4,81)$ & \\
Mixed, high & $254.0 \pm 1.5$ & $269.1 \pm 4.7$ & $*$ \\
& $(5,820)$ & $(6,303)$ & \\
P (t-test) & $* * *$ & $* * *$ & \\
\hline
\end{tabular}

Significance probabilities for $t$-test (within species):

${ }^{* * *} 0.005>\mathrm{P}>0.0005 ; * * 0.01>\mathrm{P}>0.005 ;{ }^{*} 0.025>\mathrm{P}>0.01$

0.025). Body weight of males was measured using flies from pure $D$. simulans and $D$. melanogaster bottles (Table 8). Body weight of melanogaster males was larger on standard medium as well as on wildtype yeast (significance probability for $t$-test $<$ 0.0005 ).

So, in experiment $\mathrm{D}$ we found indications that the following fitness components make D. melanogaster more successful on 'standard' medium: higher productivity, shorter duration of development and a higher body weight than simulans.

\section{Discussion}

The outcome of interspecific competition in continuous populations with overlapping generations, or with discrete generations, is known to be affected by environmental factors. Barker (in prep.) has reviewed e.g. the effects of temperature, population

Table 8. Body weight ( $\mathrm{mg} / 5 \widehat{\partial} \widehat{\delta})$ of Drosophila during experiment $D$ on wildtype yeast and standard medium $(\bar{x} \pm$ s.e. $)$.

\begin{tabular}{lll}
\hline & Wildtype yeast & Standard medium \\
\hline D. melanogaster & $4.56 \pm 0.07$ & $4.49 \pm 0.08$ \\
D. simulans & $3.78 \pm 0.05$ & $3.82 \pm 0.02$ \\
\hline Sign. prob. for t-test & $<0.0005$ & $<0.0005$
\end{tabular}


density, species frequency and medium. He suggests that "one might expect the relative competitive abilities of Drosophila species in laboratory populations to vary depending on the yeast species used in culture, and that such studies could have considerable significance in analysing the realized niches of sympatric Drosophila species in natural populations. Yet few studies have been done'.

In this respect we found in an earlier study (Bos et al., 1976) that Drosophila species differ in their abilities to colonize different environmental niches, measured by their ability to maintain themselves for five successive generations on different ergosterol mutant yeasts. This was done in the absence of interspecific competition in a medium containing solely a given strain of yeast. We found $D$. simulans superior to D. melanogaster on erg- 2 and erg- 3 yeast mutants of Saccharomyces cerevisiae. These strains contain different biological mixtures of sterols since the mutant strains used are blocked in ergosterol synthesis (Barton et al., 1974; Woods et al., 1974). What was tested were the effects of different mixtures of sterols in a biological package of yeast cells on the outcome of competition. From Cooke and Sang (1970) it is known that Drosophila has a sterol utilization similar to that of other phytophagous insects. Sterols serve a bulk requirement for which the steric criteria for utilization are relatively broad and a micronutrient requirement with a narrow steric specificity. The last requirement is only satisfied by sterols closely related in structure to cholesterol.

On standard medium, which was a killed-yeast sucrose medium, a fast extinction of simulans occurs in all experiments (Fig. 1, Table 4). This medium is clearly the most unfavourable one for simulans in a competitive situation, which is also evident from the increased development time (Table 7). Figure 2 shows that with the addition of increasing amounts of live yeast to standard medium, the competitive ability of $D$. simulans improves. There is no clear, overall difference in the final outcome of competition between the populations on mixtures of yeast strains and populations on pure wildtype yeast: in experiment A, C and D Drosophila simulans became extinct on both types of medium, whereas in experiment $\mathrm{B}$ this species outcompeted $D$. melanogaster in both types of environment. However, the data suggest that the extinction of simulans is postponed in the 'mixed' erg-3 environments

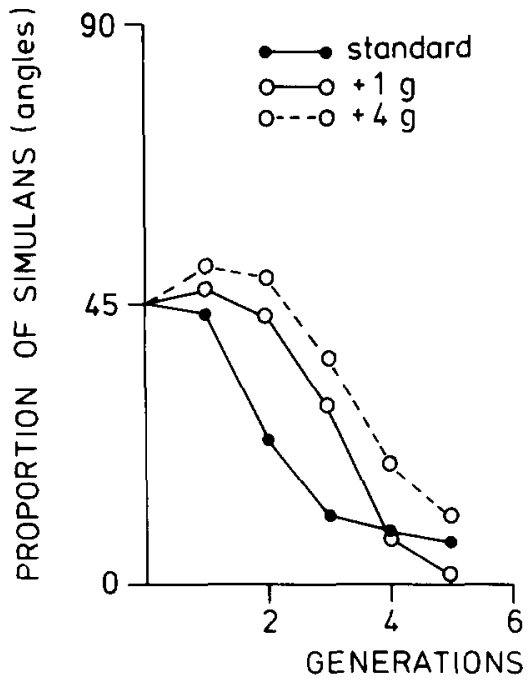

Fig. 2. Proportion of $D$. simulans in competition bottles with Standard medium enriched with different amounts of live yeast.

(Fig. 3). This or the fixation of simulans might be expected from more successful development of $D$. simulans on pure erg- 3 yeast. It was known that the amount and quality of the medium (Claringbold \& Barker, 1961; Barker, 1963) and yeast species used (El-Helw \& Ali, 1970) can significantly affect the outcome of competition between $D$. melanogaster and $D$. simulans. In our present paper we show, that even differences between yeast strains can in-

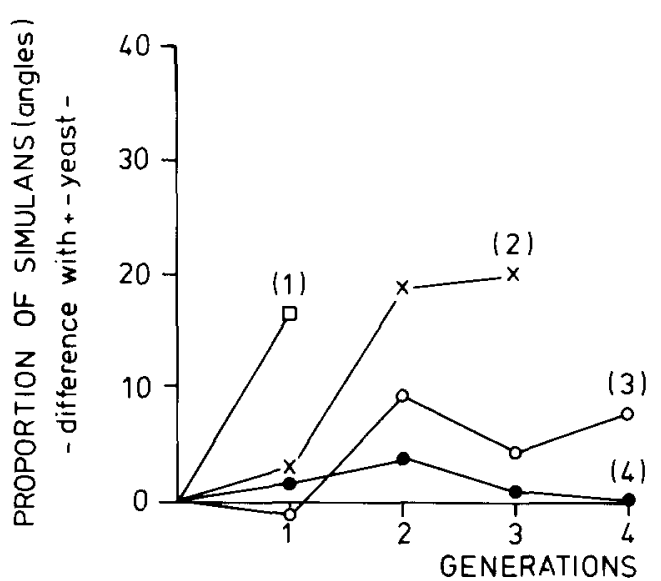

Fig. 3. Differences in proportion of simulans between environments with increased amounts of erg-3 yeast and the simultaneous controls on wildtype yeast (in angles). Standard errors of average survival are in the order of $0.9-2.1$ : (1) $100 \%$ erg-3; (2) $95 \%$ erg-3; (3) $80 \%$ erg-3; (4) $20 \% \mathrm{erg}-3$. 
fluence the competitive abilities of the two species.

It is possible to improve the competitive situation for $D$. simulans artificially by giving $D$. simulans a three-day prelay (Van Delden, 1970). Van Delden noticed an increased competitive ability only after about 77 generations of competition. So, selection for competitive ability is extremely slow, which means that the genetic component of this character is mainly non-additive (Barker, 1973; Futuyama, 1970). Therefore it is unlikely that in our experiments fixation of $D$. melanogaster or $D$. simulans within a few generations was accompanied by a selective change in the genetic components of competitive ability.

During experiments B (cages) and D (bottles) we measured productivity of females and found that the final extinction of a species in the majority of comparisons was correlated with the lowest production of adults in the competitive situation (Tables 5 and 6). In experiment $D$ we found that at least on standard medium low productivity of simulans was accompanied by long development time (Table 7) and low body weight (Table 8). This combination of fitness characters made the exclusion understandable. The addition of live yeast could postpone the exclusion of a species (Fig. 2) and the degree of postponement was dependent on the composition of the yeast (Fig. 3).

The exceptional extinction of $D$. melanogaster in the live-yeast situations in experiment $B$ seems to be caused by a temporary high productivity of the simulans females during this experiment (Table 5). This relatively higher productivity of simulans females can have had a genetic cause: the gene pool of the flies from our laboratory stock, starting the B experiment may have had features differing from the starting material of our experiments $A, C$ and $\mathrm{D}$, since several generations separated the experiments. In this case, however, it is difficult to understand that within the B experiment $D$. simulans had a relatively high productivity in the live-yeast situations and became fixed, while they had a low productivity on standard medium and became extinct. Since we know that the physiological condition of our live-yeast media can influence extremely the survival of Drosophila larvae (Bos \& Boerema, 1982) we assume an unknown environmental cause of yeast variation.

In conclusion, we think that in all our experiments differential birth rate was the effective mode of species competition. Addition of live yeast and especially mixtures of yeast strains can improve the competitive situation of a species, but can not prevent the extinction.

\section{Acknowledgements}

Our special thanks are due to Henk Mulder for drawing the figures, to Saskia Walburgh Schmidt for careful preparation of the manuscript and to Drs W. Van Delden, K. Bijlsma, B. Burnet and R. Hoekstra for helpful discussions and comments on the manuscript.

\section{References}

Barker, J. S. F., 1963. The estimation of relative fitness of Drosophila populations. II. Experimental evaluation of factors affecting fitness. Evolution 17: 56-71

Barker, J. S. F., 1973. Natural selection for coexistence or competitive ability in laboratory populations of Drosophila. Egypt. J. Genet. Cytol. 2: 288-315.

Barker, J. S. F. (in preparation). Interspecific competition. In: The genetics and biology of Drosophila. Vol. 3 ch. 37 (eds M. Ashburner and T. R. F. Wright).

Barton, D. H. R., Corrie, J. E. T., Widdowson, D. A., Bard, M. \& Woods, R. A., 1974. Biosynthesis of terpenes and steroids. IX. The sterols of some mutant yeasts and their relationship to the biosynthesis of ergosterol. J. chem. Soc. Perkin Trans. 1: 1326-1333.

Bos, M., Burnet, B., Farrow, R. \& Woods, R. A., 1976. Development of Drosophila on sterol mutants of the yeast Saccharomyces cerevisiae. Genet. Res. Camb. 28: 163-176.

Bos, M. \& Boerema, A., 1982. Drosophila and yeast conditions. Dros. Inf. Serv. 58: 26.

Claringbold, P. J. \& Barker, J. S. F., 1961. The estimation of relative fitness of Drosophila populations. J. theor. Biol. 1: 190-203.

Cooke, J. \& Sang, J. H., 1970. Utilization of sterols by larvae of Drosophila melanogaster. J. Insect Physiol. 16: 801-812.

Dobzhansky, Th., Cooper, D. M., Phaff, J. H., Knapp, E. P. \& Carson, H. L., 1956. Studies on the ecology of Drosophila in the Yosemite region of California. IV. Differential attraction of species of Drosophila to different species of yeasts. Ecology 37: 544-550.

El-Helw, M. R. \& Ali, A. M. M., 1970. Competition between Drosophila melanogaster and D. simulans on media supplemented with Saccharomyces and Schizosaccharomyces. Evolution 24: 531-537.

El-Helw, M. R., Ali, A. M. M.\& Moawad, H., 1972. Fitness of Drosophila melanogaster and $\mathrm{D}$. simulans in relation to natural genera of yeasts. Egypt. J. Genet. Cytol. 1: 196-202.

Futuyama, D. J., 1970. Variation in genetic response to interspecific competition in laboratory populations of Drosophila. Am. Nat. 104: 239-252. 
Kircher, H. W., 1969. Sterols in the leaves of the Cheirodendron gaudichandii tree and their relationship to Hawaiian Drosophila ecology. J. Insect Physiol. 15: 1167-1173.

McDonald, J. F. \& Ayala, F. J., 1974. Genetic response to environmental heterogeneity. Nature 250: 572-574.

Molzahn, S. W. \& Woods, R. A., 1972. Polyene resistance and the isolation of sterol mutants in Saccharomyces cerevisiae. J. gen. Microbiol. 72: 339-348.

Moore, J. A., 1952. Competition between Drosophila melanogaster and D. simulans 1. Population cage experiments. Evolution 6: 407-420.

Powell, J. R., 1971. Genetic polymorphism in varied environments. Science 174: 1035-1036.

Sang, J. H., 1978. The nutritional requirements of Drosophila.
In: The genetics and biology of Drosophila. Vol. 2a (eds. M. Ashburner and T. R. F. Wright).

Tantawy, A. O. \& Soliman, M. H., 1967. Studies on natural populations of Drosophila. VI. Competition between Drosophila melanogaster and D. simulans. Evolution 21: 34-40.

Van Delden, W., 1970. Selection for competitive ability. Dros. Inf. Serv, 45: 169.

Woods, R. A., Bard, M., Gardner, I. E. \& Molzahn, S. W., 1974. Studies on the accumulation of ergosterol and 24(28)-dehydroergosterol in 3 strains of Saccharomyces cerevisiae. Microbios 10A: 73-80.

Received 13. 9. 1982; Accepted 1. 2. 1983. 